December 1999

\title{
Educational Policy: Egalitarian or Elitist?
}

\author{
Herschel I. Grossman \\ Brown University \\ Minseong Kim \\ University of Pittsburgh
}

\begin{abstract}
This paper offers an explanation for observed differences across countries in educational policies and in resulting interpersonal distributions of human capital. We analyse a generalequilibrium model in which, as a result of the apportionment of natural ability, nurturing, and publicly financed education, some people can be well endowed with human capital, whereas other people are poorly endowed with human capital. We assume that people can choose to be either producers or predators. Because an increase in a person's human capital makes predation a less attractive choice for that person, it is possible that by using some of their human capital to educate the poorly endowed people the well endowed people can increase their own consumption. More interestingly, our theory predicts that, if producers are able to enforce a collective choice that takes advantage of the deterrent effect of allocating resources to guarding against predators, then the well endowed people prefer a relatively egalitarian educational policy that increases the human capital of all of the poorly endowed people. Such an educational policy either decreases the cost of deterring predation or makes deterrence possible. In contrast, if producers or small subsets of producers individually choose the amount of their resources to allocate to guarding, taking the ratio of predators to producers as given, then the well endowed people prefer an elitist educational policy that, if it has a redistributional component, decreases the number of poorly endowed people, thereby decreasing the number of predators, without increasing the human capital of the remaining poorly endowed people. These implications seem to be consistent with the facts about differences across countries in educational policy.
\end{abstract}

We acknowledge helpful comments from Murat Iyigun, Juan Mendoza, and Suk Jae Noh. Arsène Aka gave us valuable assistance with the data. 
Mr. Benson looks to the future and sees a widening social abyss. "We could get to a situation similar to Manila, where the upper and middle classes surround themselves with walls and security guards to protect themselves from an abandoned underclass." ... When asked about Mr. Benson's fear of an urban America that looks like Manila, Mr. Newsome shrugs: "It's already like that," he says. (The Wall Street Journal, September 26, 1995, page A8.)

Although almost every modern state provides either public education or public subsidies for education, casual observation reveals that differences in educational policy result in large differences across countries in the interpersonal distribution of human capital. Most notably, in Western European countries and in some East Asian countries, like Japan, Korea, Singapore, and Taiwan, every person receives a good enough education to insure that almost everyone is at least functionally literate. In these countries the resulting distribution of human capital is relatively egalitarian. In contrast, in many of the countries of South America, Africa, and Asia, including the Philippines, and, as Mr. Newsome suggests, also in the United States, educational policy is elitist. In these countries, while many people receive higher education and acquire a large amount of human capital, many other people receive little or no effective education and do not even achieve functional literacy. These people constitute "an abandoned underclass" to which Mr. Benson refers.

This paper proposes a positive theory of the redistributional component of publicly financed education. The theory suggests an explanation for why educational policy is egalitarian in some countries but elitist in other countries.

Our analytical framework is a general-equilibrium model in which, as a result of the apportionment of natural ability, nurturing, and publicly financed education, some people can be well endowed with human capital, whereas other people are poorly endowed with human capital. In this model the interpersonal distribution of human capital has two dimensions. One dimension is the endowment of human capital of a poorly endowed person relative to a 
well endowed person. The other dimension is the fraction of people who are poorly endowed.

If educational policy has a redistributional component, then the well endowed are using some of their human capital to educate people who, because of either modest natural ability or ineffective nurturing or both, are initially poorly endowed with human capital. An egalitarian educational policy has a redistributional component that increases the human capital of all of the poorly endowed people. An elitist educational policy, in contrast, may or may not have a redistributional component. Also, if it has a redistributional component, then an elitist educational policy only decreases the number of poorly endowed people without increasing the human capital of the remaining poorly endowed people.

To theorize about educational policy we focus on the predatory threat posed by people who are poorly endowed with human capital. To analyse this predatory threat we model people as choosing to be either producers or predators, predators being people who produce nothing, but live by appropriating the product of the producers.

We assume that each person chooses to be either a producer or a predator depending on whether production or predation is more lucrative for him. ${ }^{1}$ We also assume that being a predator is a more attractive choice for a poorly endowed person than for a well endowed person. To implement this assumption as simply as possible, we specify technologies such that a person's human capital enhances his ability to produce, but does not enhance his effectiveness as a predator. ${ }^{2}$ Given these technologies, and given that human capital is

\footnotetext{
${ }^{1}$ Dan Usher (1987) developed a pioneering general-equilibrium model in which, as in the present model, people choose whether to be producers or predators and producers decide how much time and effort to put into guarding against predators. In another paper - Grossman and Kim (2000) - we analyse the choices of people to be producers or predators in a model in which only some people, whom we define to be amoral, are potential predators. The other people, whom we define to be moral, always choose to be producers, no matter how lucrative predation is relative to production. Usher (1997) assumes that education can increase the fraction of people who are moral. In the present paper we assume that everybody is amoral. Assuming that some people are moral would not change the main predictions about educational policy.

${ }^{2}$ This assumption implies that our analysis is applicable to low-skilled predatory activities like burglary,
} 
inalienable and can be neither directly consumed nor directly exchanged for consumables, a person benefits from a larger endowment of human capital only by choosing to be a producer.

The existence of poorly endowed people who might choose to be predators negatively affects the well endowed people in two ways: First, producers allocate some of their potentially productive human capital to guarding against predators. ${ }^{3}$ Second, unless predation is deterred, producers lose some of their production to predators. These negative effects of predation connect to educational policy because the well endowed people can mitigate the threat of predation by using some of their human capital to educate those people who are initially poorly endowed with human capital. ${ }^{4,5}$

robbery, and kidnapping. We implicitly abstract from high-skilled predatory activities like embezzlement and some forms of litigation.

${ }^{3}$ Guarding against predators includes all actions that are costly but have the effect of decreasing the fraction of production lost to predation. Examples of ways of guarding against predators include the locating of production in inconvenient but secure places, the production of things that are harder for predators to appropriate, the installation of locks, the building of walls, the hiring of private security guards, and the organizing of a police force. For simplicity, our analysis focuses only the total amount of resources allocated to guarding, abstracting from different ways of guarding. Further, although the model does not explicitly consider the apprehension and punishment of predators, we could easily extend the analysis to allow for apprehension and punishment. The apprehension and punishment of predators would not directly decrease the ability of predators to appropriate the product of producers, but apprehension and punishment by decreasing the expected utility of predators would make the choice to be a predator less attractive.

${ }^{4}$ We could apply a similar analysis to any redistributive policy from which a person can benefit only by choosing not to be a predator. For example, Grossman (1994) considered the possibility that the optimal response of a propertied class to the threat of predation could be a redistribution of land, whereas Grossman (1995) considered the possibility of a subsidy to wages to induce people to choose to be producers. Neither of these earlier papers addressed the central issue in the present paper, which is the interaction between guarding against predators and redistributive policy.

${ }^{5}$ Our model abstracts from externalities in production. As many authors have shown, such externalities can induce well endowed people to subsidize the education of poorly endowed people, even in the absence of a predatory threat. For example, François Bourguignon and Thierry Verdier (1996) emphasize production 
Our model assumes that the well endowed people make a collective choice of this redistributional component of educational policy. ${ }^{6}$ In addition, our model allows the educational policy that the well endowed people choose to be either egalitarian or elitist, or some combination of egalitarian and elitist. An egalitarian educational policy makes production more lucrative for all of the poorly endowed people, and thereby decreases the amount of guarding against predators that is necessary to deter poorly endowed people from choosing to be predators. An elitist educational policy, if it has a redistributional component, decreases the number of people for whom predation is more lucrative than production, and thereby decreases the number of people who choose to be predators.

In addition to distinguishing between countries with egalitarian and elitist educational policies, we also distinguish countries in which the decision to allocate resources to guarding against predators is made individually from countries in which this decision is made collectively. In our theory an individual producer, or a small subset of producers, who chooses the amount of his human capital to allocate to guarding takes the choices of other people to be either predators or producers as given. In contrast, a collective choice of the amount of guarding takes into account the deterrent effect of guarding on the number of people who choose to be predators.

Although this strategic advantage of a collective choice of the amount of guarding is externalities in a model in which, in contrast to the present model, education enhances the ability of poorly endowed people to pose a political threat to well endowed people. In the present model well endowed people have an incentive to educate poorly endowed people only to mitigate the predatory threat from the poorly endowed people. This simplification focuses our analysis on explaining why educational policy is egalitarian in some countries but elitist in other countries.

${ }^{6}$ We regard this view that donors take the initiative in redistributive policy to be both more general and more realistic, even in "democracies", than the popular median-voter model of redistribution. Also, we implicitly assume that the cost of receiving a publicly financed education, which would include the opportunity cost of time spent in school, is small enough that an initially poorly endowed person would accept any offer of publicly financed education. 
important, the enforcement of collective choices is always problematic. ${ }^{7}$ In exploring the effect of the limited ability of producers to enforce a collective choice of the amount of guarding, we consider three possible cases.

In the first case, the limited ability of producers to enforce a collective choice of the amount of guarding against predators does not impose a binding constraint. In the second case, the limited ability of producers to enforce a collective choice of the amount of guarding imposes a binding constraint, but with an appropriate redistributive educational policy this constraint is not tight enough to negate the strategic advantage of collective choice. In the third case, the producers' ability to enforce a collective choice of the amount of guarding is so limited that the strategic advantage of collective choice is lost. In this third case producers, or small subsets of producers, individually choose the amount of guarding.

Casual observation suggests that Western European countries and some East Asian countries, like Japan, Korea, and Singapore, and Taiwan, provide historical examples in which producers collectively choose the amount of guarding against predators. Interestingly, we have already observed that these same countries have relatively egalitarian educational policies that provide even the poorly endowed with a relatively good education. In contrast, other countries, mainly in South America, Africa, and Asia, including the Philippines and, perhaps, also the United States, provide historical examples in which at the margin producers, or small subsets of producers, individually choose the amount of guarding. Even more interestingly, we have observed that these countries have elitist educational policies that give some people an excellent education and other people little or no education. These examples suggest that egalitarian educational policies are associated with collective choice of guarding, whereas elitist educational policies are associated with individual choice of guarding.

\footnotetext{
${ }^{7}$ To be more concrete, we can think of government as being the agent to whom producers assign the task of enforcing a collective choice of the amount of guarding, with taxation being the means of enforcement. In this context limited ability to enforce a collective choice of the amount of guarding reflects limited ability to collect taxes from producers.
} 
To complement this anecdotal evidence we computed the following cross-country regressions:

(I) hcsd $=0.66+0.28 \mathrm{ELF}$

(14.9) (3.3)

65 Observations $\quad R^{2}=0.14 \quad$ t-statistics in parentheses

(II)

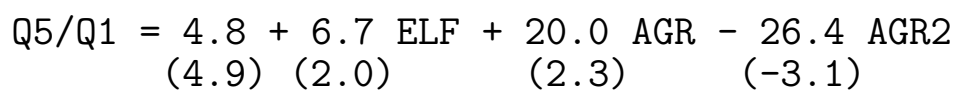

73 Observations $\quad R^{2}=0.11 \quad$ t-statistics in parentheses

In regression (I) the dependent variable, hesd, is the standard deviation of the logarithm of $(1+$ years of schooling $)$ for people older than 15 years. We take this variable to be a measure of inequality in the distribution of human capital. ${ }^{8}$ In regression (II) the dependent variable, Q5/Q1, is the ratio of the income of the top quintile to the income of the bottom quintile in the income distribution. We take this variable to be an alternative measure of inequality in the distribution of human capital. ${ }^{9}$ The regressor, ELF, is a measure of the degree of ethnic diversity. Following William Easterly and Ross Levine (1997) and Alberto Alesina, Reza Baqir, and Easterly (1997), who suggest that difficulties in providing public goods are attributable to political polarization caused by ethnic diversity, we use ELF as a proxy for limited ability of producers to enforce a collective choice of the amount of guarding. ${ }^{10}$ The other regressors, AGR and AGR2, are the fraction of labor force in the agricultural

\footnotetext{
${ }^{8}$ The source for hcsd was Josè De Gregorio and Jong-Wha Lee (1999). We used the observation for the year 1990 .

${ }^{9}$ The source for Q5/Q1 was Klaus Deininger and Lyn Squire (1996). We used the observation closest to the year 1990. The use of the Gini coefficient as an alternative proxy measure for inequality in the distribution of human capital yielded almost identical results.

${ }^{10} \mathrm{ELF}$ is an acronym for ethno-linguistic fractionalization. The source for ELF was Anthony Annett (1997) and David Barrett (1992), whose data provides a more detailed and more recent breakdown of ethnolinguistic groups than does the data on ethnic diversity used by Paulo Mauro (1995) and by Easterly and Levine (1997).
} 
sector and the square of this fraction. These variables control for the level of economic development. ${ }^{11}$ These regressions show positive and statistically significant dependence of both hcsd and Q5/Q1 on ELF. A possible inference from these regressions is that collective choice of guarding is associated with an egalitarian educational policy, whereas individual choice of guarding is associated with an elitist educational policy, just as the anecdotal evidence suggests. ${ }^{12}$

Our theoretical analysis provides an explanation for these associations. We find that, if producers collectively choose the amount of guarding against predators, then the well endowed people prefer an egalitarian educational policy that increases the human capital of all of the poorly endowed people and, thereby, decreases the amount of guarding that is necessary to deter poorly endowed people from choosing to be predators. In contrast, if producers individually choose the amount of guarding against predators, then the well endowed people prefer an elitist educational policy that, if it has a redistributional component, decreases the number of poorly endowed people and, thereby, decreases the number of people who choose to be predators.

\section{Analytical Framework}

Let $K$ denote the human capital of each well endowed person, and let $k$ denote the human capital of each poorly endowed person, where $K \geq k>0$. Let $U$ denote the ratio of poorly endowed people to well endowed people. The fraction of people who are poorly endowed equals $U /(1+U)$, and the fraction of people who are well endowed equals

\footnotetext{
${ }^{11}$ The source for AGR was World Bank (1993). We used the observation for 1990. Together the positive coefficient for AGR and the negative coefficient for AGR2 capture the Kuznets curve.

${ }^{12}$ As additional support for the anecdotal evidence, we found for 14 OECD countries a positive correlation of 0.5 between ELF and an internationally comparable measure of inequality in human capital, defined as the difference between the scores of the 75 th percentile and the 25 th percentile in each country on an international test of mathematics and science for primary school students. The source of this measure of inequality in human capital was OECD (1997).
} 
$1 /(1+U)$. Aside from their endowments of human capital, people are otherwise identical. The average endowment of human capital, denoted by $\Omega$, is

$$
\Omega=\frac{K+U k}{1+U}
$$

Let $e$, where $0 \leq e<1$, denote the fraction of his human capital that each well endowed person allocates to educating people who are initially poorly endowed with human capital. The variable $e$ captures the redistributional component of publicly financed education. ${ }^{13}$ Assuming that each unit of human capital allocated to education produces one additional unit of human capital, $\Omega$ is related to the average initial endowment of human capital, denoted by $\Omega_{o}$, according to

$$
\Omega=\Omega_{o}+\frac{e K}{1+U} .
$$

Equating these two expressions for $\Omega$ and rearranging, we can relate $(1-e) K$, the amount of human capital that each well endowed person has available for the production of consumables, to $\Omega_{o}, U$, and $k$. Specifically,

$$
(1-e) K=\Omega_{o}+U\left(\Omega_{o}-k\right)
$$

Equation (1) implies that, for a given value of $\Omega_{o}$, the combination of $U$ and $k$ fully describes the interpersonal distribution of human capital available for the production of consumables. ${ }^{14}$

\footnotetext{
${ }^{13}$ This formulation restricts educational policy to be in effect a redistribution of human capital from the well endowed to the initially poorly endowed. This restriction accords with our concern with whether or not the initially poorly endowed are "an abandoned underclass". In a different context Raquel Fernandez and Richard Rogerson (1995) suggest that partial public funding of higher education can involve a redistribution from poorer to richer people.

${ }^{14}$ If each unit of human capital allocated to education produces $\lambda$ additional units of human capital, where $\lambda$ is positive but not necessarily equal to one, then equation (1) generalizes to $(1-\lambda e) K=\Omega_{o}+U\left(\Omega_{o}-k\right)$. All of the qualitative conclusions derived below obtain whether or not $\lambda$ equals one.
} 
Given the amount of human capital that he has available for production each person must choose whether to be a producer or a predator. Let $N$ denote the fraction of people who are well endowed and who choose to be producers, let $n$ denote the fraction of people who are poorly endowed and who choose to be producers, and let $R$ denote the ratio of predators to producers. The fraction of people, whether well endowed or poorly endowed, who choose to be predators equals $R /(1+R)$, which is identical to $1-N-n$.

If a person chooses to be a producer, then he must allocate his human capital between production and guarding against predators. Let $G$ denote the ratio of the human capital that a producer allocates to guarding against predators to the human capital that he allocates to the production of consumables. The fraction of his human capital that a producer allocates to guarding equals $G /(1+G)$, and the fraction of his human capital that a producer allocates to production equals $1 /(1+G)$.

As discussed above, the choice of $G$ can be made either collectively or individually. Let $\bar{G}$ denote the maximum collective choice of $G$ that producers can enforce. The present analysis takes $\bar{G}$ to be an exogenous variable. Endogenizing $\bar{G}$ would be an interesting extension.

To simplify the analysis of the choice between being a producer or a predator, assume that a unit of human capital can produce one unit of consumables. The number of units of consumables that a producer actually produces equals the product of the amount of human capital that he has available for production and the fraction of this human capital that he allocates to production. Thus, a well endowed producer produces $(1-e) K /(1+G)$ units of consumables, a poorly endowed producer produces $k /(1+G)$ units of consumables, and, with the same $G$ for all producers, total production equals $[N(1-e) K+n k] /(1+G)$ units of consumables.

Let $p$ denote the fraction of his production that a producer appropriates. Predators 
appropriate the fraction $1-p \cdot{ }^{15}$ It is natural to assume that $p$ depends negatively on the ratio of predators to producers and positively on a producer's guarding ratio. ${ }^{16}$ Specifically, assume that

$$
p=\frac{1}{1+\theta R / G}, \text { where } \theta \geq 0
$$

In equation (2), the parameter $\theta$, which embodies the technology of predation, determines the effectiveness of predators in appropriating consumables for given values of $R$ and $G$. The specification that $p$ does not depend on the identity of the predators reflects the assumption that well endowed people and poorly endowed people are equally effective at predation. $^{17}$

Let $C$ denote the consumption of a well endowed producer, and let $c$ denote the consumption of a poorly endowed producer. As a well endowed producer produces (1 e) $K /(1+G)$ units of consumables, and a poorly endowed producer produces $k /(1+G)$

\footnotetext{
${ }^{15}$ For simplicity the model abstracts from possible destruction of some consumables as the result of predation. In Grossman and Kim (1995) we show how destruction is easily incorporated into the analysis.

${ }^{16} \mathrm{~A}$ random matching story provides a simple rationale for these assumptions. The larger is the ratio of predators to producers the more frequently will each producer encounter a predator. The larger is the ratio of resources allocated to guarding against predators to production that has to be guarded the less successful will the predators be in each encounter.

${ }^{17}$ Equation (2) assumes, for simplicity, that for each producer $p$ depends only on $R$ and on his own guarding ratio. We could extend the model to allow for a negative externality in guarding. For example, it is possible that, if your neighbors build high walls around their properties but you do not build a high wall around your property, then your property becomes a relatively easier target for burglars. In this case, for each producer $p$ also would be positively related to his own guarding ratio relative to the guarding ratio of other producers. It is easy to show that in the case of individual choice of the amount of guarding this effect would cause each producer to choose a larger guarding ratio for any given value of $R$. But, because individual producers take $R$ as given, a negative externality in guarding would not change the conclusion that in the case of collective choice of the amount of guarding producers, taking into account the deterrent effect of guarding, would choose to enforce an even larger guarding ratio.
} 
units of consumables, we have

$$
C=\frac{p(1-e) K}{1+G}
$$

and

$$
c=\frac{p k}{1+G}
$$

Let $D$ denote the consumption of a predator. As total production equals $[N(1-e) K+$ $n k] /(1+G)$ units of consumables, assuming that each predator obtains an equal share of the total amount of consumables appropriated from the producers, we have

$$
D=\frac{1-p}{R /(1+R)} \frac{N(1-e) K+n k}{1+G}
$$

According to equation (5) $D$ is a decreasing function of $R$. If $R$ equals zero, then the analysis takes the value of $D$ to be $\lim _{R \rightarrow 0} D$, which, using equations (1) and (2), equals $\theta \Omega_{o} / G(1+G)$.

\section{The Choice to be a Producer or a Predator}

To decide whether to be a producer or a predator, each well endowed person compares the values of $C$ and $D$, both of which he takes as given, and each poorly endowed person compares the values of $c$ and $D$, both of which he takes as given. Using equations (1) (5) we find that the choices of well endowed and poorly endowed people to be producers or predators depend on $G$ in the following way: ${ }^{18}$

1. If $D$ equals $C$ but is larger than $c$, then poorly endowed people prefer to be predators, whereas well endowed people are indifferent between being producers or predators. This case would occur if and only if $G$ equals $\theta$. In this case, $R$ can take any value larger than or equal to $U$.

\footnotetext{
${ }^{18}$ The ratios $D / C$ and $D / c$ are undefined for $G$ smaller than $\theta$.
} 
2. If $D$ is smaller than $C$ but larger than $c$, then poorly endowed people prefer to be predators, whereas well endowed people prefer to be producers. This case could occur only if $G$ is larger than $\theta$, but smaller than $\theta(1-e) K / k$. In this case, $R$ is equal to $U$.

3. If $D$ is smaller than $C$ but equal to $c$, then poorly endowed people are indifferent between being producers or predators, whereas well endowed people prefer to be producers. This case could occur only if $G$ is equal to or larger than $\theta \Omega_{o} / k$, but not larger than $\theta(1-e) K / k$. In this case, the equality between $D$ and $c$ implies that $R$ is equal to $\left(G-\theta \Omega_{o} / k\right) /\left(\theta \Omega_{o} / k-\theta\right)$. This implied value of $R$ is equal to or smaller than $U$, but larger than or equal to zero.

4. If $D$ is smaller than both $c$ and $C$, then every person prefers to be a producer. This case could occur only if $G$ is larger than $\theta \Omega_{o} / k$. In this case, $R$ equals zero.

Summarizing these results we have

$$
R=\left\{\begin{array}{lrl}
x \in[U, \infty] & \text { if and only if } & G=\theta \\
U & \text { only if } & \theta<G<\theta(1-e) K / k \\
\frac{G-\theta \Omega_{o} / k}{\theta \Omega_{o} / k-\theta} & \text { only if } & \theta \Omega_{o} / k \leq G \leq \theta(1-e) K / k \\
0 & \text { only if } & G>\theta \Omega_{o} / k .
\end{array}\right.
$$

The piecewise linear locus in Figure 1 illustrates equation (6). The next section turns to the choice of $G$ by producers.

\section{The Ratio of Predators to Producers with Unconstrained Collective Choice of the Amount of Guarding}

Consider a country in which the limited ability of producers to enforce a collective choice of the amount of guarding against predators, as represented by $\bar{G}$, does not impose a binding constraint. In this country the producers collectively choose $G$ to maximize $C$ and $c$, as given by equations (3) and (4). This unconstrained collective choice of $G$ takes 
into account both the effect of $G$ on $p$ for a given ratio of predators to producers, as given by equation (2), and the effect of $G$ on the choices of well endowed people and poorly endowed people to be predators, as given by equation (6). Because a producer choosing his amount of guarding individually would not take into account the effect of $G$ on $R$, the unconstrained collective choice of $G$ is as large as or larger than producers would choose individually. Hence, given an unconstrained collective choice of $G$ producers would not individually choose to allocate any additional human capital to guarding.

Substituting equations (2) and (6) into equations (3) and (4), we find that both $C$ and $c$ have a local maximum at $G$ equal to $G^{*}$, where $G^{*}$ equals $(1+\epsilon) \theta(1-e) K / k$, and where $\epsilon$, a small positive number, is the smallest fraction by which the producers collectively can choose to increase $G$. From equation (6), $G^{*}$ is the minimum value of $G$ that would result in an equilibrium in which $R$ uniquely equals zero - that is, the minimum value of $G$ that would surely deter every person, whether well endowed or poorly endowed, from choosing to be a predator. ${ }^{19}$ Note that $G^{*}$ is positively related to $(1-e) K / k$.

We also find that both $C$ and $c$ can have either one of two other local maxima. One of these possible local maxima is at $G$ equal to $\sqrt{\theta U}$, which is the solution to the firstorder conditions for an interior maximum, $d C / d g=d c / d g=0$, given $R=U$. The other possible local maximum is at $G$ equal to $(1+\epsilon) \theta$, which is the minimum value of $G$ that would deter only all of the well endowed people from choosing to be predators. Note that $(1+\epsilon) \theta$ is also the value that $G^{*}$ approaches as $k$ approaches $(1-e) K$ - that is, as the interpersonal distribution of human capital becomes more egalitarian.

To determine the global maximum, we compare the values of $C$ and $c$ associated with $G$ equal to $G^{*}, \sqrt{\theta U}$, and $(1+\epsilon) \theta$. Substituting equations (2) and (6) into equation (3)

\footnotetext{
${ }^{19}$ Equation (6) says that $R=0$ could also be associated with values of $G$ such that $\theta(1-e) K / k \geq$ $G>\theta \Omega_{o} / k$. But, such values of $G$ also can be associated with positive values of $R$.
} 
we find that the values of $C$ that would result from each of these possible choices of $G$ are

$$
C= \begin{cases}\frac{(1-e) K}{1+G^{*}} & \text { for } G=G^{*} \\ \frac{(1-e) K}{(1+\sqrt{\theta U})^{2}} & \text { for } G=\sqrt{\theta U} \\ \frac{(1-e) K}{\left(1+\frac{U}{1+\epsilon}\right)[1+(1+\epsilon) \theta]} & \text { for } G=(1+\epsilon) \theta\end{cases}
$$

The values of $c$ that would result from each of these possible choices of $G$ are the same, except that $k$ replaces $(1-e) K$ in the numerator of each expression. Given $k$ and $U$ the producers collectively choose the value of $G$ for which the resulting values of $C$ and $c$ are largest. Denote the resulting value of $C$ as $C_{J}$.

Equation (7) implies that either the smaller is $(1-e) K / k$ or the larger is $U$ the more likely are the producers collectively to set $G$ equal to $G^{*}$ in order to deter all people, both the poorly endowed as well as the well endowed, from choosing to be predators. The intuition for this result is that the smaller is $(1-e) K / k$ the smaller is $G^{*}$, whereas the larger is $U$ the larger would be the ratio of predators to producers and the resulting fraction

of production lost to predation if the poorly endowed people were not deterred from choosing to be predators.

\section{Educational Policy With Unconstrained Collective Choice of the Amount of Guarding}

Let $k_{o}$ denote the initial endowment of human capital of an initially poorly endowed person, let $K_{o}$ denote the initial endowment of human capital of a well endowed person, and let $U_{o}$ denote the ratio of initially poorly endowed people to initially well endowed people. Equation (1) implies that $K_{o}$ equals $\Omega_{o}+U_{o}\left(\Omega_{o}-k_{o}\right)$. To make the problem interesting assume that initial interpersonal distribution of human capital is such that the initial ratio of poorly endowed to well endowed people is large and that initially poorly 
endowed people have little human capital relative to the well endowed people. Specifically, assume that $k_{o}$ is smaller than $K_{o} \sqrt{\theta / U_{o}}$ and that $U_{o}$ is larger than $\theta$. Given this initial interpersonal distribution of human capital both $C$ and $c$ have local maxima at $G$ equal to $(1+\epsilon) \theta K_{o} / k_{o}$ and at $G$ equal to $\sqrt{\theta U_{o}}$. Either one or the other of these local maxima can be the global maximum. Figure 2 illustrates how $C$ depends on $G$ in this initial situation.

As discussed above, the well endowed people make a collective choice of the fraction of their human capital to allocate to educating people who are initially poorly endowed with human capital. In addition, the educational policy that the well endowed people collectively choose can be either egalitarian, in that it increases the human capital of all of the poorly endowed people, or elitist, in that, if it has a redistributional component, it only decreases the number of poorly endowed people, or some combination of egalitarian and elitist. The objective of the well endowed people is to maximize their own consumption. ${ }^{20}$

The problem that the well endowed people solve is to choose $k$ and $U$ to maximize $C_{J}$, the consumption of a well endowed person, as derived from equation (7), subject to $k \geq k_{o}$ and $U \leq U_{o}$. The constraints $k \geq k_{o}$ and $U \leq U_{o}$ are consistent with educational policy being in effect a redistribution of human capital from the well endowed to the initially poorly endowed. Equation (1) implies that, given $\Omega_{o}$, the choice of $k$ and $U$ also determines $(1-e) K$, the amount of human capital that each well endowed person has available for the production of consumables.

Substituting equation (1) into equation (7) we find that, with $U$ larger than $\theta$, the

\footnotetext{
${ }^{20}$ For ease of exposition we analyse sequentially the three decisions - the choice of each person to be a producer or a predator, the choice by producers of the amount of guarding against predators, and the choice by well endowed people of an educational policy. The equilibrium of our model simultaneously reconciles these choices. In this equilibrium the consumption of the people with the largest consumption is maximized. In Grossman and Kim (1999) we use a model similar to the model in the present paper to derive the distribution of human capital that maximizes the consumption of the person with the smallest consumption.
} 
maximum value of $C_{J}$ obtains for $G$ equal to $G^{*}$, the minimum value of $G$ for which $R$ equal to zero unambiguously obtains, and for $k /(1-e) K$ equal to $\sqrt{\theta / U}$. In addition, for these values of $G$ and $k /(1-e) K$ the maximum value of $C_{J}$ is a nondecreasing function of $U$. Thus, with $U_{o}$ larger than $\theta$ and $k_{o}$ smaller than $K_{o} \sqrt{\theta / U_{o}}$, the solution to the problem of maximizing $C_{J}$ is for the initially well endowed people to leave $U$ unchanged and equal to $U_{o}$, but to increase $k$, and to decrease $(1-e) K$, to satisfy

$$
k=(1-e) K \sqrt{\theta / U_{o}}
$$

Increasing $k$ and decreasing $(1-e) K$ decreases $G^{*}$.

Given this interpersonal distribution of human capital, the producers collectively choose

$$
G=G^{*}=(1+\epsilon) \sqrt{\theta U_{o}}
$$

From equation (9) we see that the assumption that $\bar{G}$ is not a binding constraint implies that $\bar{G}$ is at least as large as $(1+\epsilon) \sqrt{\theta U_{o}}$. (See Mathematical Appendix A for a more complete derivation of these results.)

This analysis says that, if the limited ability of producers to enforce a collective choice of the amount of guarding against predators does not impose a binding constraint, then the well endowed people choose an egalitarian educational policy that increases the human capital of all of the initially poorly endowed people. Figure 3 illustrates how $C$ depends on $G$ with this egalitarian educational policy. This educational policy, however, does not equalize everyone's human capital, nor does it decrease the fraction of people who are poorly endowed. Given the interpersonal distribution of human capital that results from this educational policy, the producers collectively chose enough guarding against predators to deter everyone from choosing to be a predator. $^{21}$ In this case, the educational policy

\footnotetext{
${ }^{21}$ This implication involves the implicit assumption that every person who is initially poorly endowed with human capital is sufficiently educable to be able to achieve the endowment $k$ as given by equation (8). In reality, it is likely that some people are not sufficiently educable to achieve this endowment of human
} 
that the well endowed people choose balances the marginal benefit of decreasing the cost of deterring predation by increasing the human capital of poorly endowed people with the marginal cost of increasing the human capital of poorly endowed people.

\section{Educational Policy and The Ratio of Predators to Producers with Con- strained Collective Choice of the Amount of Guarding}

Consider another country in which the limited ability of producers to enforce a collective choice of the amount of guarding against predators, as represented by $\bar{G}$, imposes a binding constraint. Specifically, assume that $\bar{G}$ is smaller than $(1+\epsilon) \sqrt{\theta U_{o}}$, which is the choice of $G$ derived in the preceding section, but also assume that $\bar{G}$ is at least as large as $(1+\epsilon) \theta$. Recall that $(1+\epsilon) \theta$ is the minimum value of $G$ that would deter all of the well endowed people from choosing to be predators, and that it is also the value that $G^{*}$ approaches as $k$ approaches $(1-e) K$. In this country the well endowed people collectively choose the educational policy that maximizes $C$, given that the producers collectively choose $G$ to maximize $C$ and $c$, subject to the constraint $G \leq \bar{G}{ }^{22}$

Substituting equation (1) into equation (7) we find that, with $U$ larger than $\theta$, the maximum value of $C_{J}$, subject to the constraint $G \leq \bar{G}$, obtains for $k /(1-e) K$ equal to $(1+\epsilon) \theta / \bar{G}$ and for $G$ equal to $\bar{G}$. With $k /(1-e) K$ equal to $(1+\epsilon) \theta / \bar{G}, \bar{G}$ is

capital. The choice of $G$ as given by equation (9) would not deter these insufficiently educable people from choosing to be predators. Accordingly, we should not take the implication that everyone is deterred from predation literally. We should expect that in reality, even with unconstrained collective choice of the amount of guarding and a educational policy that attempts to increase everyone's human capital to $k$ as given by equation (8), some insufficiently educable people choose to be predators.

${ }^{22}$ This assumption implies that, although the limited ability of producers to enforce a collective choice of the amount of guarding against predators imposes a binding constraint, the well endowed people do not face a binding constraint on their ability to enforce their collective choice of an educational policy. A possible rationale for this assumption is that the collective choice of educational policy involves only the well endowed people, whereas the collective choice of the amount of guarding is more difficult because it involves all of the producers, who can include poorly endowed people in addition to well endowed people. 
equal to $G^{*}$. In addition, for these values of $k /(1-e) K$ and $G$ the maximum value of $C_{J}$ is a nondecreasing function of $U$.

Thus, with $U_{o}$ is larger than $\theta$ and $k_{o}$ smaller than $K_{o} \sqrt{\theta / U_{o}}$, the solution to the problem of maximizing $C_{J}$ is for the well endowed people to leave $U$ unchanged and equal to $U_{o}$, but to increase $k$, and to decrease $(1-e) K$, to satisfy

$$
k=(1-e) K(1+\epsilon) \theta / \bar{G} .
$$

Given this interpersonal distribution of human capital, the producers collectively choose

$$
G=\bar{G}=G^{*}
$$

(See Mathematical Appendix B for a more complete derivation of these results.)

This analysis says that, given that the limited ability of producers to enforce a collective choice of the amount of guarding against predators imposes a binding constraint, but that producers can enforce a collective choice of guarding that is at least large enough to deter all of the well endowed people from choosing to be predators, the well endowed people choose an egalitarian educational policy that increases the human capital of all of the initially poorly endowed people sufficiently to make it possible, by setting $G$ equal to $\bar{G}$, to deter everyone from choosing to be a predator. ${ }^{23}$ Given this educational policy, the producers collectively chose the maximum amount of guarding against predators that they can enforce, and everyone is deterred from choosing to be a predator, as in the previous case in which $\bar{G}$ was not a binding constraint.

\footnotetext{
${ }^{23}$ Equation (10) implies that $k /(1-e) K$ is an increasing function of $\bar{G}$. As $\bar{G}$ approaches $(1+\epsilon) \theta$, $k /(1-e) K$ must approach one to make it possible to deter everyone from choosing to be a predator. In this case the educational policy that the well endowed people choose might come close to equalizing everyone's human capital.
} 


\section{The Ratio of Predators to Producers with Individual Choice of the Amount of Guarding}

Finally, consider a country in which $\bar{G}$, the maximum collective choice of $G$ that producers can enforce, is smaller than $(1+\epsilon) \theta$. In this country not only is $\bar{G}$ a binding constraint, but, as we shall see, $\bar{G}$ is not larger than the guarding ratio that producers would choose individually. Hence, in this country the strategic advantage of collective choice of guarding is lost.

In individually choosing the amount of guarding, each producer takes the choices of other people to be producers or predators as given. Thus, if a well endowed person chooses to be a producer, then he chooses $G$ to maximize $C$, taking $R$ as given, and if a poorly endowed person were to choose to be a producer, then he would choose $G$ to maximize $c$, taking $R$ as given.

To analyse the individual choice of $G$ we substitute equation (2) into equations (3) and (4) and calculate the value of the ratio $G$ that satisfies the conditions $d C / d g=0$ and $d c / d g=0$. For both well endowed producers and poorly endowed producers this ratio is given by

$$
G=\sqrt{\theta R} .
$$

Solving equations (6) and (12) for $R$, and assuming that the ratio $k / \Omega_{0}$ is small relative to $\theta$, we find that the equilibrium ratio of predators to producers with individual choice of $G$ is

$$
R=\max \{\theta, U\} .
$$

Equation (13) says that, if $U$, the ratio of poorly endowed people to well endowed people, is equal to or larger than $\theta$, then all of the poorly endowed people choose to be predators, and all of the well endowed people choose to be producers. Figure 4 illustrates the solution of equations (6) and (12) for $U$ larger than $\theta$. Alternatively, if $U$ were smaller than $\theta$, 
then all of the poorly endowed people and some of the well endowed people would choose to be predators. In this equilibrium well endowed people would be indifferent between being producers and being predators. Figure 5 illustrates the solution of equations (6) and (12) for $U$ smaller than $\theta .^{24}$

With well endowed people either preferring to be producers or being indifferent between being producers and being predators the consumption of a well endowed person is equal to $C$. Substituting for $p$ from equation (2) and for $G$ and $R$ from equations (12) and (13) into equation (3), we calculate the equilibrium value of $C$, denoted $C_{I}$, to be

$$
C_{I}= \begin{cases}\frac{(1-e) K}{(1+\theta)^{2}} & \text { for } \quad U \leq \theta \\ \frac{(1-e) K}{(1+\sqrt{\theta U})^{2}} & \text { for } \quad U>\theta .\end{cases}
$$

Equation (14), together with equation (1), tells us how $C_{I}$ depends on $k$ and $U$.

\section{Educational Policy Given Individual Choice of the Amount of Guarding}

Assume again that the well endowed people make a collective choice of the fraction of their human capital to allocate to educating people who, because of either modest natural ability or ineffective nurturing or both, are initially poorly endowed with human capital. With individual choice of the amount of guarding the problem that the well endowed people

${ }^{24}$ More generally, solving equations (6) and (12) for $R$ would yield

$$
R= \begin{cases}\theta & \text { for } \quad U \leq \theta \\ U & \text { for } \quad \theta<U \leq R_{1} \\ R_{1} & \text { for } \quad R_{1}<U<R_{2} \\ \left\{R_{1}, R_{2}, U\right\} & \text { for } U \geq R_{2},\end{cases}
$$

where $R_{1}$ and $R_{2}$ are the values of $R$ that satisfy both $R=\left(G-\theta \Omega_{o} / k\right) /\left(\theta \Omega_{o} / k-\theta\right)$, from equation (6), and $G=\sqrt{\theta R}$, from equation (12). Such values of $R$ would exist only if $k / \Omega_{0}$ were sufficiently large relative to $\theta$. 
solve is to choose $k$ and $U$ to maximize $C_{I}$, as given by equation (14), subject to $k \geq k_{o}$ and $U \leq U_{o}$. Assume again that $U_{o}$ is larger than $\theta$.

Substituting equation (1) into equation (14) we find that $C_{I}$ is a decreasing function of $k$. This result obtains because increasing $k$ would decrease $(1-e) K$ without affecting $R$. Thus, part of the solution to the problem of maximizing $C_{I}$ is for the well endowed people to leave $k$ unchanged and equal to $k_{o}$.

We also find that, for $U$ smaller than $\theta, C_{I}$ is an increasing function of $U$. This result obtains because, as $R$ cannot be smaller than $\theta$, making $U$ even smaller that $\theta$ would decrease $(1-e) K$ without affecting $R$.

Finally, we find that, for $U$ larger than $\theta, C_{I}$ is a quasi-convex function of $U$. Specifically, $C_{I}$ has a local maximum at $U$ equal to $\theta$, has a local minimum at a value of $U$ that is larger than $\theta$, and approaches a finite limit from below as $U$ goes to infinity. If and only if $U$ equal to $\theta$ is not the global maximum, then there exists a value of $U$, denoted $\hat{U}$, such that $C_{I}$ evaluated at any value of $U$ larger than $\hat{U}$ is larger than $C_{I}$ evaluated at $U$ equal to $\theta$. (Note that $\hat{U}$, if it exists, is larger than $\theta$.) Figures 6 and 7 depict the relation between $C_{I}$ and $U$ on the assumption that $\hat{U}$ exists.

This analysis implies that the other part of the solution to the problem of maximizing $C_{I}$ may or may not call for the well endowed people to use some of their human capital to decrease $U$. If $\hat{U}$ exists and if $U_{o}$ is as large as or larger than $\hat{U}$, as illustrated in Figure 6 , then the well endowed people leave $U$ unchanged and equal to $U_{o}$. In Figure 6 we see that decreasing $U$ from $U_{o}$ could only decrease $C_{I}$. In this case, the well endowed people do not choose to use any of their human capital to educate people who are initially poorly endowed. This result obtains because, with a small fraction of people who are initially well endowed, a given decrease in $U$ would require that a large fraction of the human capital of the well endowed people be used for education.

Alternatively, either if $\hat{U}$ does not exist or if $U_{o}$ is smaller than $\hat{U}$, as illustrated in Figure 7, then to maximize $C_{I}$ the well endowed people decrease $U$ to make $U$ 
equal $\theta$. In Figure 7 we see that $U$ equal to $\theta$ would yield the largest value of $C_{I}$ for all values of $U$ less than or equal to $U_{o}$. In this case, the well endowed people choose to educate some of the initially poorly endowed people sufficiently to make them also well endowed, thereby decreasing the fraction of people who are poorly endowed, but leaving the remaining poorly endowed people with no more than their initial endowment of human capital. This elitist educational policy reduces $R$, the ratio of predators to producers, from $U_{o}$ to $\theta$. Because of the producers' limited ability to enforce a collective choice of the amount of guarding, educational policy is directed towards decreasing the number of predators rather than facilitating the deterrence of predation.

\section{Summary}

Assuming that people can choose to be either producers or predators, we have found that well endowed people can maximize their own consumption by choosing collectively to use some of their human capital to educate people who, because of modest natural ability or ineffective nurturing or both, are initially poorly endowed with human capital. More importantly, we found that whether the well endowed people choose an egalitarian educational policy that increases the human capital of all of the poorly endowed people or an elitist educational policy that, if it has a redistributional component, only decreases the number of poorly endowed people without increasing the human capital of the remaining poorly endowed people depends on the ability of producers to enforce a collective choice of the amount of guarding against predators.

We considered three possible cases. In the first case, the limited ability of producers to enforce a collective choice of the amount of guarding does not impose a binding constraint. In the second case, the limited ability of producers to enforce a collective choice of the amount of guarding imposes a binding constraint, but this constraint is not tight enough to negate the strategic advantage of collective choice in taking into account the deterrent effect of guarding on the number of people who choose to be predators. In the third case, 
the producers' ability to enforce a collective choice of the amount of guarding is so limited that the strategic advantage of collective choice is lost.

We found that in the first two cases the well endowed people maximize their own consumption by choosing an egalitarian educational policy that by increasing the human capital of all of the poorly endowed people either decreases the cost of deterring predation or makes deterrence possible. In contrast, in the third case producers, or small subsets of producers, individually choose the amount of human capital to allocate to guarding. Importantly, an individual producer, or small subset of producers, takes the choices of other people to be either predators or producers as given, ignoring the deterrent effect of guarding on the number of people who choose to be predators. We found that in this case the well endowed people maximize by their own consumption by choosing an elitist educational policy that, if it has a redistributional component, decreases the number of poorly endowed people and, thereby, decreases the number of predators.

Applying this theory, we contrast two sets of countries. One set includes Western European countries and some East Asian countries, like Japan, Korea, Singapore, and Taiwan, that seem to provide historical examples of collective choice of the amount of guarding against predators, and in which, as a result, the amount of guarding has been sufficient to deter most, if not all, low-skilled predatory activities like burglary, robbery, and kidnapping. Our theory leads us to expect these countries to have egalitarian educational policies that provide even relatively poorly endowed people with a good education. The other set includes countries in South America, Africa, and Asia, including the Philippines, and, perhaps, also the United States, that seem to provide historical examples of individual choice of the amount of guarding against predators. Our theory leads us to expect these countries to have elitist educational policies that give some people an excellent education and other people little or no effective education. As we have noted, these implications seem to be consistent with the facts about differences across countries in educational policy. 


\section{Mathematical Appendices}

\section{A. Educational Policy Given Unconstrained Collective Choice of the Amount of} Guarding

Let $C_{J}^{*}$ denote the global maximum of $C_{J}$ subject to the constraints $k_{o} \leq k \leq \Omega_{o}$ and $0 \leq U \leq U_{o}$. We find the values of $k$ and $U$ associated with $C_{J}^{*}$ by comparing the three possible local maxima, as given by equation (7), each maximized with respect to $U$ and $k$.

1. Substituting equation (1) into the first row of equation (7), we find that the local maximum for $C$ at $G=(1+\epsilon) \theta(1-e) K / k$ is maximized with $U=U_{o}$ and

$$
k=\min \left\{\frac{\sqrt{1+\epsilon}\left(1+U_{o}\right)}{U_{o}+\sqrt{U_{o} / \theta}}, 1\right\} \Omega_{o}
$$

or, equivalently, $k /(1-e) K=\min \left\{\sqrt{\theta / U_{o}}, 1\right\}$. The resulting value of $C$, denoted $C^{I}$, is

$$
C^{I}= \begin{cases}\frac{\Omega_{o}}{1+(1+\epsilon) \theta} & \text { for } \quad U_{o} \leq \theta \\ \frac{\Omega_{o}\left(1+U_{o}\right)}{\left(1+\sqrt{\theta U_{o}}\right)\left[1+\sqrt{(1+\epsilon) \theta U_{o}}\right]} & \text { for } \quad U_{o}>\theta .\end{cases}
$$

2. Substituting equation (1) into the second row of equation (7), we find that the local maximum of $C$ at $G=\sqrt{\theta U}$, if it exists, is maximized either with $U=(1+\epsilon) \theta$ and $k=k_{o}$ or with $U=U_{o}$ and $k=k_{o}$. The resulting value of $C$, denoted $C^{I I}$, is either

$$
C^{I I}=\frac{[1+(1+\epsilon) \theta] \Omega_{o}-(1+\epsilon) \theta k_{o}}{(1+\theta \sqrt{1+\epsilon})^{2}} \text { with } U=(1+\epsilon) \theta \text { and } k=k_{o}
$$

or

$$
C^{I I}=\frac{\left(1+U_{o}\right) \Omega_{o}-U_{o} k_{o}}{\left(1+\sqrt{\theta U_{o}}\right)^{2}} \text { with } U=U_{o} \text { and } k=k_{o} .
$$

3. Substituting equation (1) into the third row of equation (7), we find that the local maximum of $C$ at $G=(1+\epsilon) \theta$, if it exists, is maximized with $U=0$. The resulting maximized value of $C$, denoted $C^{I I I}$, is $C^{I I I}=\Omega_{o} /[1+(1+\epsilon) \theta]$. 
We can easily see that, if $U_{o}>\theta$, then $C^{I}>C^{I I}$ and $C^{I}>C^{I I}$, regardless of whether $C^{I I}$ occurs with $U=(1+\epsilon) \theta$ or $U=U_{o}$. Thus, $C_{J}^{*}$ is associated with $U=U_{o}$ and with $k$ and $G$ as given by equations (8) and (9).

\section{B. Educational Policy With Constrained Collective Choice of the Amount of Guarding}

Let $\bar{C}_{J}$ denote the global maximum of $C_{J}$ subject to the constraint $G \leq \bar{G}$ and subject to the constraints $k_{o} \leq k \leq \Omega_{o}$, and $0 \leq U \leq U_{o}$. Assume that $U_{o}>\theta$ and $(1+\epsilon) \theta \leq \bar{G}<(1+\epsilon) \sqrt{\theta U_{o}}$. Again, we find the values of $k$ and $U$ associated with $\bar{C}_{J}$ by comparing the three possible local maxima, as given by equation (7), each maximized with respect to $U$ and $k$.

1. Although the value of $G$ associated with the maximization of the first row of equation (7) with respect to $U, k$, and $G$ does not satisfy the constraint $G \leq \bar{G}$, by substituting equation (1) into $G=(1+\epsilon) \theta(1-e) K / k$ and rewriting the constraint $G \leq \bar{G}$ as $(1+\epsilon) \theta\left[\Omega_{o}+U\left(\Omega_{o}-k\right)\right] / k \leq \bar{G}$, we see that the binding constraint of $\bar{G}$ can be satisfied at $G=(1+\epsilon) \theta(1-e) K / k$ either by decreasing $U$ or by increasing $k$. Because both decreasing $U$ and increasing $k$ reduces the value of $C$ at $G=(1+\epsilon) \theta(1-e) K / k$ from the unconstrained maximum, the constrained maximum would occur with the constraint $G \leq \bar{G}$ satisfied as an equality. Substituting $G=\bar{G}=(1+\epsilon) \theta(1-e) K / k$ into the first row of equation (7), we find that, with the constraint $G \leq \bar{G}$ satisfied as an equality, the local maximum for $C$ at $G=(1+\epsilon) \theta(1-e) K / k$ is $C=k \bar{G} /(1+\epsilon) \theta(1+\bar{G})$. This local maximum for $C$ is maximized with the largest value of $k$ that satisfies the constraints $U \leq U_{o}, k \leq \Omega_{o}$, and $G=\bar{G}$. Thus, this local maximum for $C$ is maximized with $U=U_{o}$ and

$$
k=\frac{(1+\epsilon) \theta\left(1+U_{o}\right) \Omega_{o}}{(1+\epsilon) \theta U_{o}+\bar{G}}
$$

or, equivalently, $k=(1-e) K(1+\epsilon) \theta / \bar{G}>\sqrt{\theta / U_{o}}$. The resulting value of $C$, denoted $\bar{C}^{I}$, 
is

$$
\bar{C}^{I}=\frac{\bar{G}\left(1+U_{o}\right) \Omega_{o}}{(1+\bar{G})\left[(1+\epsilon) \theta U_{o}+\bar{G}\right]} .
$$

2. Similarly, although $G=\sqrt{\theta U_{o}}$ does not satisfy the constraint $G \leq \bar{G}$, this constraint can be satisfied by decreasing $U$. In this case, because decreasing $U$ decreases the value of $C$ at $G=\sqrt{\theta U}$ from the unconstrained maximum, the constrained maximum would occur with the constraint $G \leq \bar{G}$ satisfied as an equality. The value of $G$ associated with $U=(1+\epsilon) \theta$ satisfies the constraint $G \leq \bar{G}$. Substituting $G=\bar{G}=\sqrt{\theta U}$ and equation (1) into the second row of equation (7), we find that, with the constraint $G \leq \bar{G}$ satisfied as an equality, the local maximum for $C$ at $G=\sqrt{\theta U}$ is maximized either with $U=(1+\epsilon) \theta$ and $k=k_{o}$ or with $U=\bar{G}^{2} / \theta$ and $k=k_{o}$. The resulting value of $C$, denoted $\bar{C}^{I I}$, is either

$$
\bar{C}^{I I}=\frac{[1+(1+\epsilon) \theta] \Omega_{o}-(1+\epsilon) \theta k_{o}}{(1+\theta \sqrt{1+\epsilon})^{2}} \text { with } U=(1+\epsilon) \theta \text { and } k=k_{o}
$$

or

$$
\bar{C}^{I I}=\frac{\left(1+\bar{G}^{2} / \theta\right) \Omega_{o}-\bar{G}^{2} k_{o} / \theta}{(1+\bar{G})^{2}} \text { with } U=\bar{G}^{2} / \theta \text { and } k=k_{o} .
$$

3. The constraint $G \leq \bar{G}$ is not binding for the maximization of the local maximum for $C$ at $G=(1+\epsilon) \theta$. As in the previous section, the local maximum for $C$ at $G=(1+\epsilon) \theta$, if it exists, is maximized with $U=0$ and $C^{I I I}=\Omega_{o} /[1+(1+\epsilon) \theta]$.

We can easily confirm that $\bar{C}^{I}>\bar{C}^{I I}$ and $\bar{C}^{I}>C^{I I}$, regardless of whether $\bar{C}^{I I}$ occurs with $U=(1+\epsilon) \theta$ or $U=\bar{G}^{2} / \theta$. Thus, $\bar{C}_{J}$ is associated with $U=U_{o}$ and $k$ and $G$ as given by equations (10) and (11). 


\section{References}

Alesina, Alberto, Baqir, Reza, and Easterly, William. "Public Goods and Ethnic Divisions", unpublished, March 1997.

Annett, Anthony M. "Ethnic and Religious Division, Political Instability, and Government Consumption", unpublished, November 1997.

Barrett, David B., ed. World Christian Encyclopedia : A Comparative Study of Churches and Religions in the Modern World, AD 1900-2000, New York: Oxford University Press, 1982.

Bourguignon, François and Verdier, Thierry. "Oligarchy, Democracy, Inequality, and Growth," unpublished, November 1966.

Deininger, Klaus and Squire, Lyn. "A New Data Set Measuring Income Inequality", World Bank Economic Review, 10, September 1996, 565-591.

De Gregorio, Josè and Lee, Jong-Wha. "Education and Income Distribution: New Evidence From Cross-Country Data", unpublished, 1999.

Easterly, William and Levine, Ross. "Africa's Growth Tragedy: Policies and Ethnic Divisions", Quarterly Journal of Economics, 112, November 1997, 1203-1250.

Fernandez, Raquel and Rogerson, Richard. "On the Political Economy of Education Subsidies", Review of Economic Studies, 62, April 1995, 249-262.

Grossman, Herschel I. "Production, Appropriation, and Land Reform," American Economic Review, 84, June 1994, 705-712.

Grossman, Herschel I. "Robin Hood and the Redistribution of Property Income," European Journal of Political Economy, 11, September 1995, 399-410.

Grossman, Herschel I. and Kim, Minseong. "Swords or Plowshares? A Theory of the Security of Claims to Property," Journal of Political Economy, 103, December 1995, 1275-1288.

Grossman, Herschel I. and Kim, Minseong. "Predation, Efficiency, and Inequality," unpub- 
lished, July 1999.

Grossman, Herschel I. and Kim, Minseong. "Predators, Moral Decay, and Moral Revivals," European Journal of Political Economy, forthcoming, 2000.

Mauro, Paolo. "Corruption and Growth", Quarterly Journal of Economics, 110, August 1995, 681-712.

OECD. Education at a Glance: OECD Indicators, 1997.

Usher, Dan. "Theft as a Paradigm for Departures from Efficiency," Oxford Economic Papers, 39, June 1987, 235-252; reprinted as Chapter III in Dan Usher, The Welfare Economics of Markets, Voting and Predation, Ann Arbor: The University of Michigan Press, 1992.

Usher, Dan. "Education As a Deterrent to Crime," Canadian Journal of Economics, 30, May 1997, 367-384.

World Bank, Social Indicators of Development, 1993. 


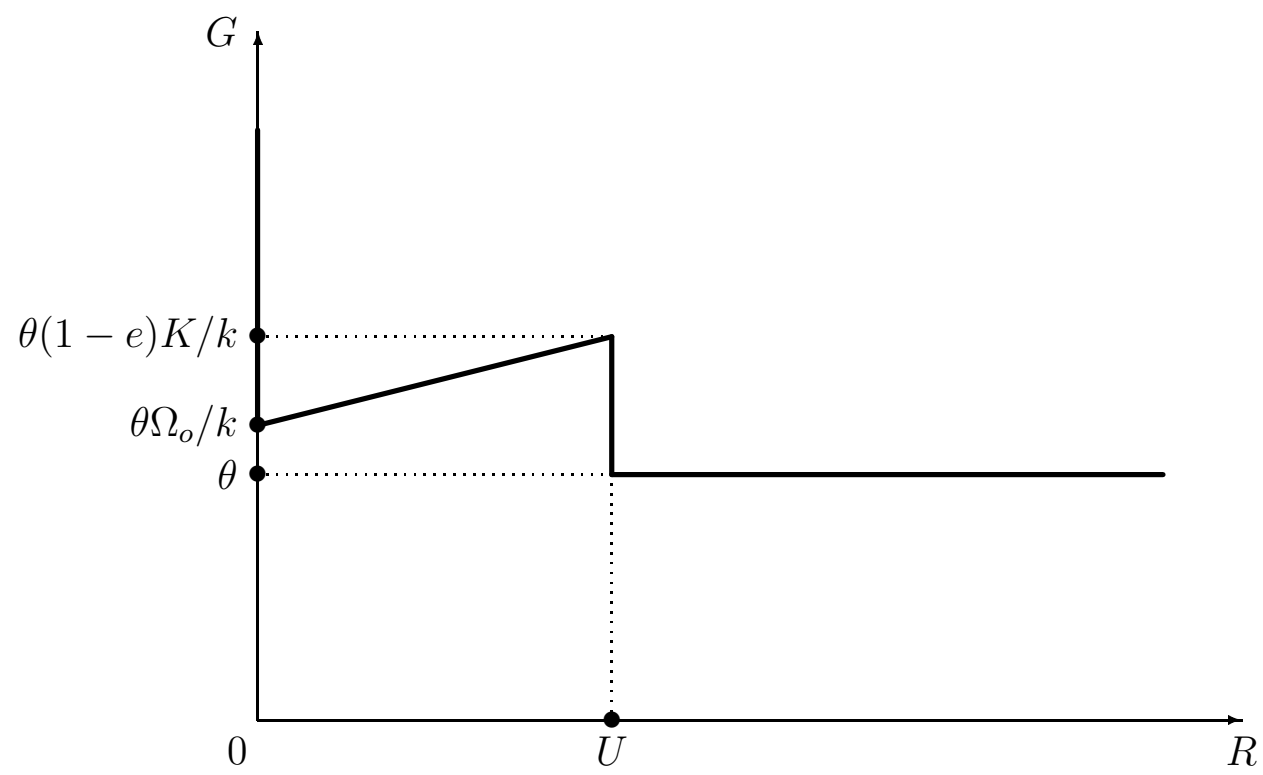

Figure 1: The Choice to be a Producer or a Predator 


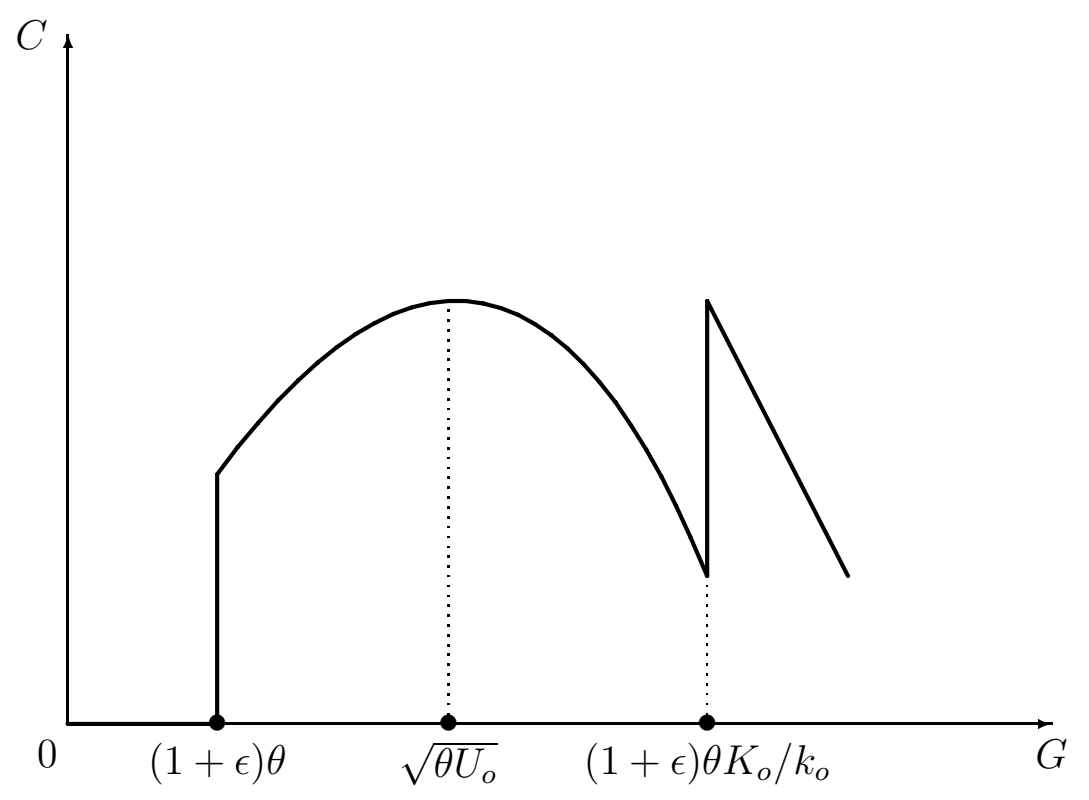

Figure 2: Guarding and Consumption with the Initial Interpersonal Distribution of Human Capital 


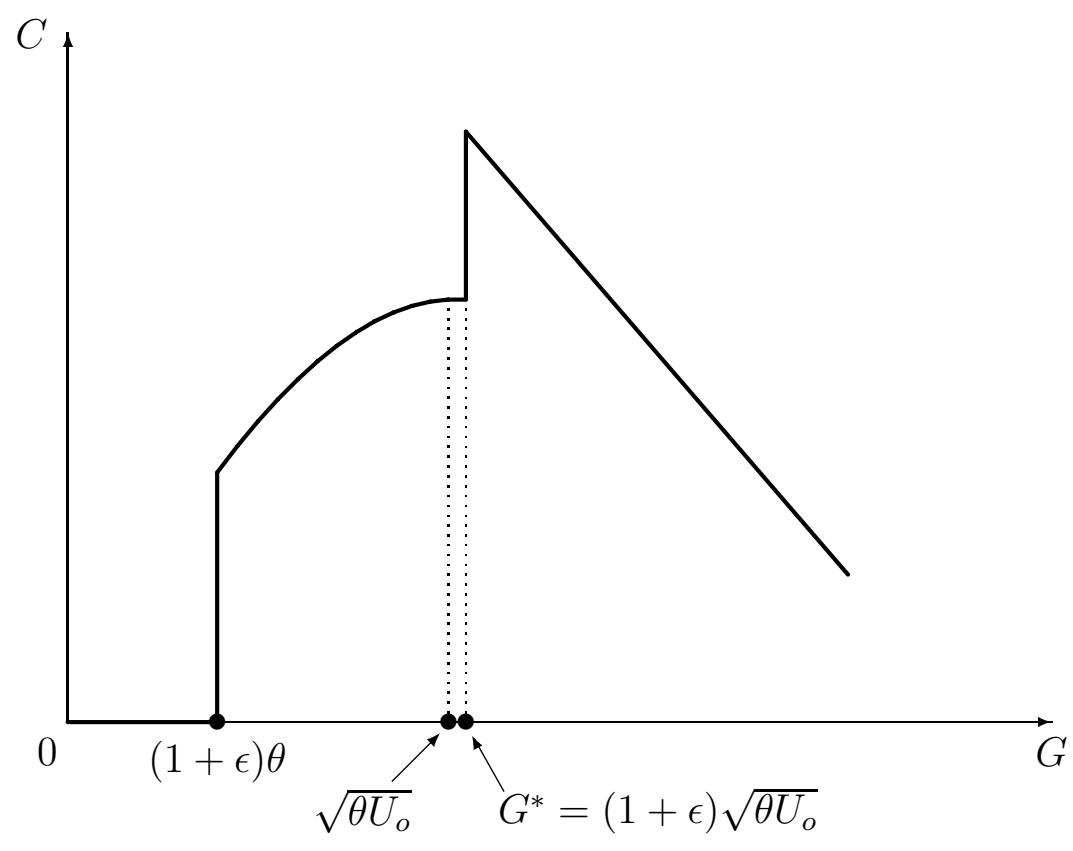

Figure 3: Guarding and Consumption with an Egalitarian Educational Policy 


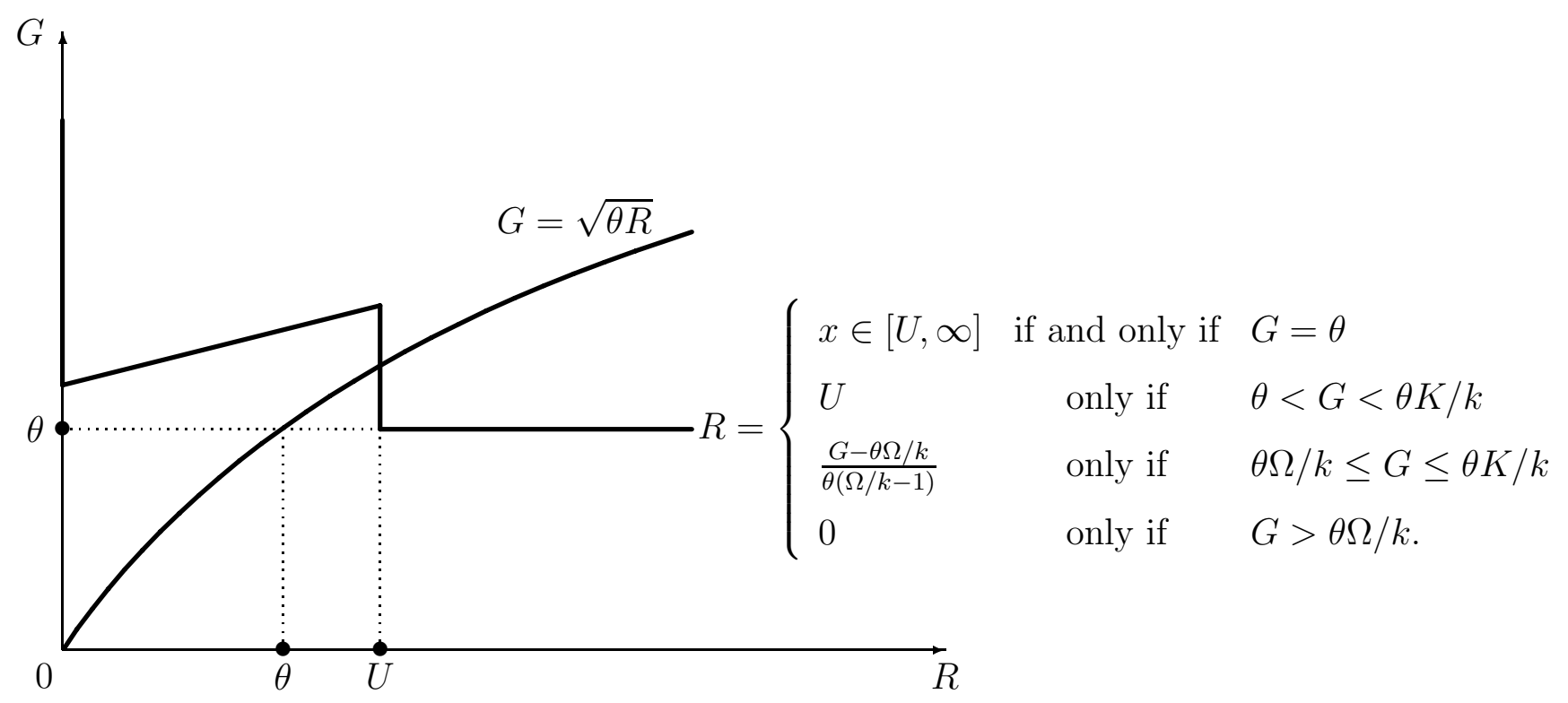

Figure 4: Individual Choice of Guarding with $R=U>\theta$

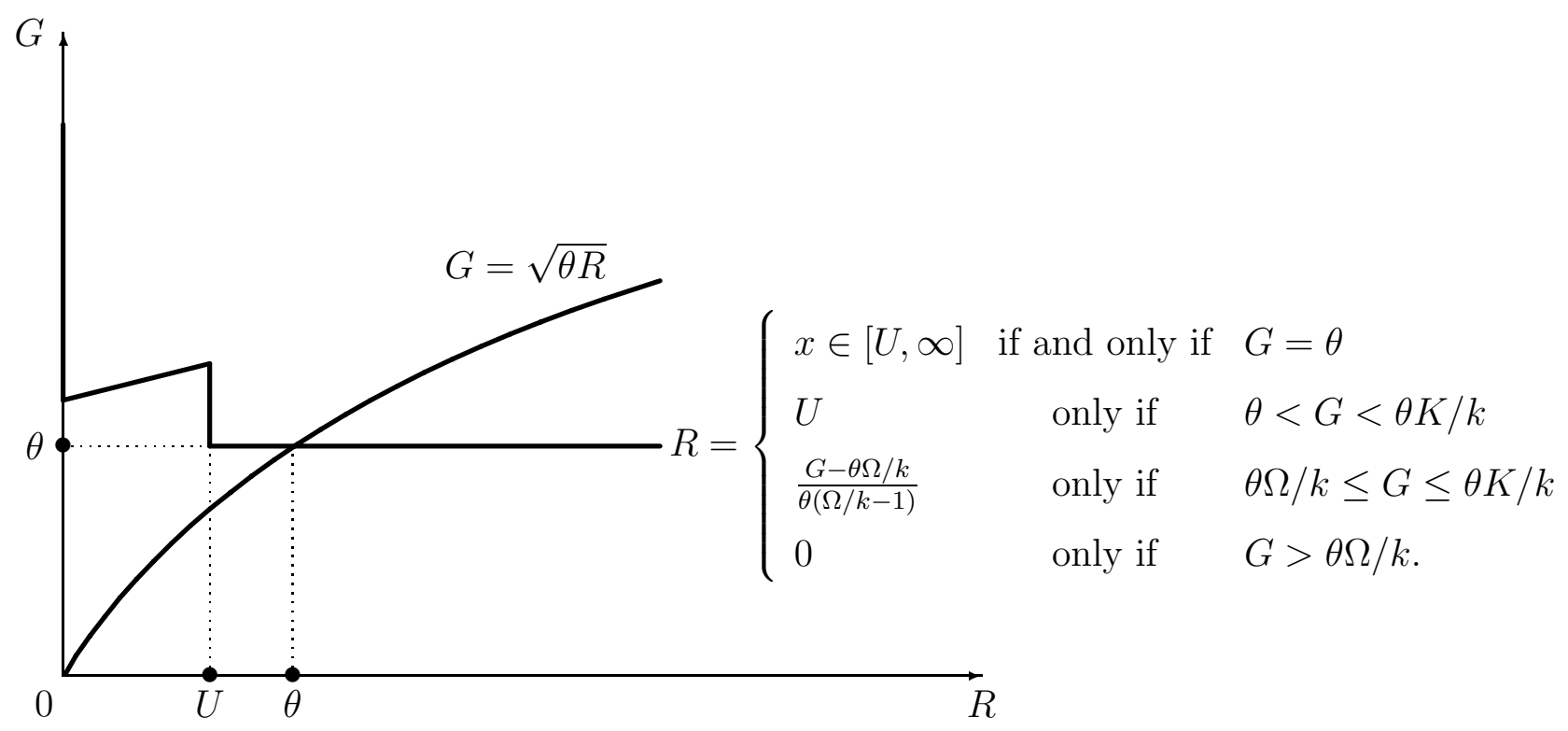

Figure 5: Individual Choice of Guarding with $R=\theta>U$ 


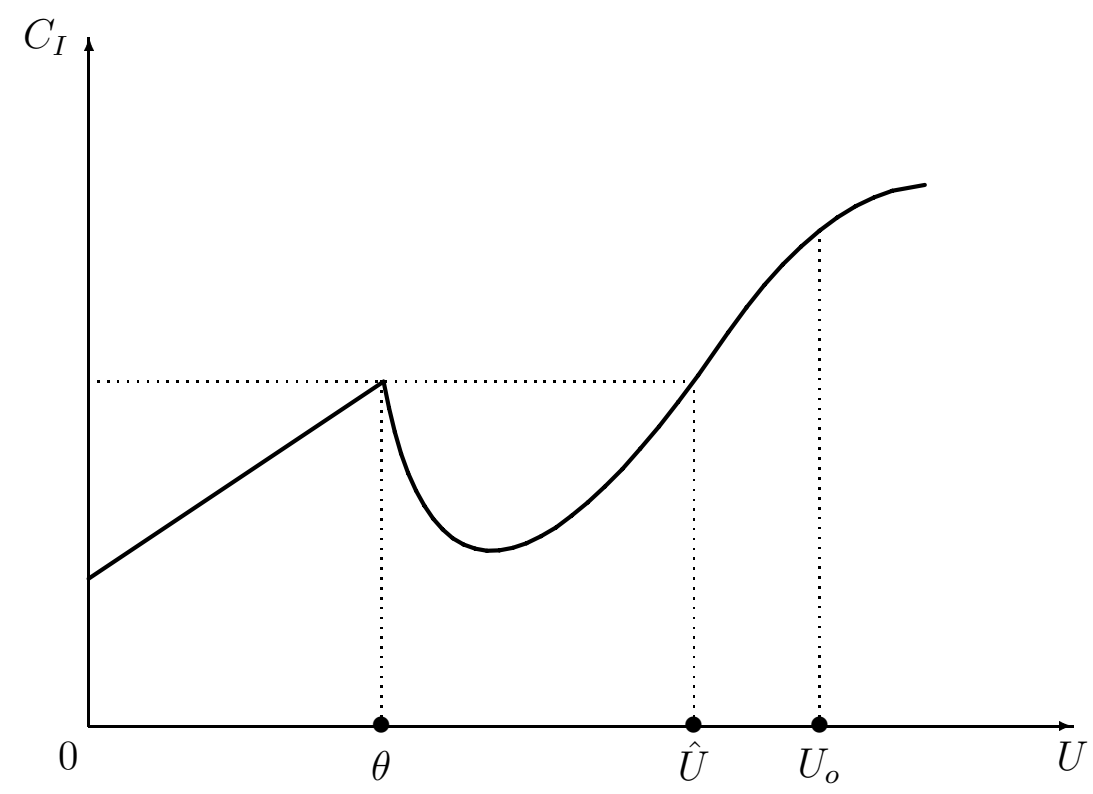

Figure 6: $U_{o}>\hat{U} \Rightarrow$ No Educational Policy

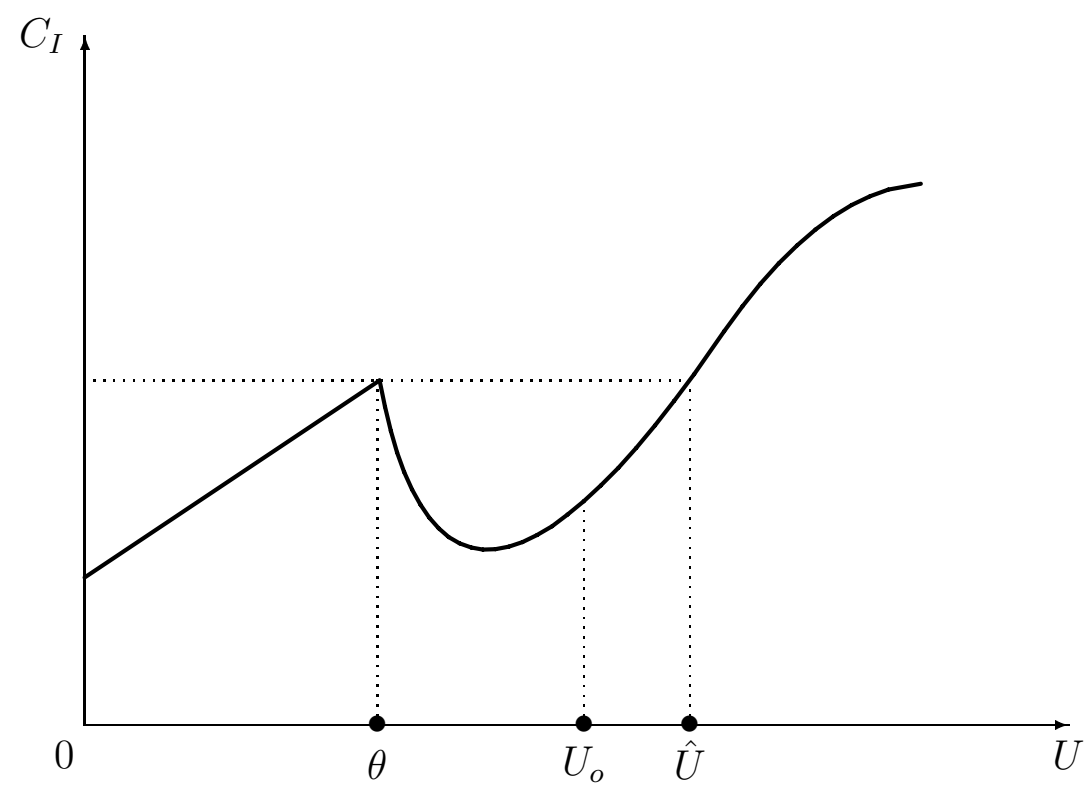

Figure 7: $U_{o}<\hat{U} \Rightarrow$ Elitist Educational Policy 